\title{
Silencing of CDCA5 inhibits cancer progression and serves as a prognostic biomarker for hepatocellular carcinoma
}

\author{
JIANLIN WANG $^{1 *}$, CONGCONG XIA $^{1 *}$, MENG PU $^{2 *}$, BIN DAI $^{1}$, XISHENG YANG $^{1}$, RUNZE SHANG $^{1}$, \\ ZHEN YANG ${ }^{1}$, RUOHAN ZHANG ${ }^{1}$, KAISHAN TAO ${ }^{1}$ and KEFENG DOU ${ }^{1}$ \\ ${ }^{1}$ Department of Hepatobiliary Surgery, Xijing Hospital, The Fourth Military Medical University, Xi'an, Shaanxi 710032; \\ ${ }^{2}$ Department of Hepatobiliary Surgery, The Air Force General Hospital of \\ The People's Liberation Army, Beijing 100142, P.R. China
}

Received March 12, 2018; Accepted July 9, 2018

DOI: $10.3892 /$ or.2018.6579

\begin{abstract}
Cell division cycle associated 5 (CDCA5) has been associated with the progression of several types of cancers. However, its possible role and mechanism in hepatocellular carcinoma (HCC) remain unknown. In the present study, immunohistochemical staining and real-time PCR were used to assess CDCA5 protein and mRNA levels in clinical samples. Statistical analysis was performed to explore the clinical correlation between CDCA5 protein expression and clinicopathological features and overall survival in HCC patients. Cell counting and colony formation assays were employed to analyse the effect of CDCA5 on cell proliferation, and flow cytometry was used to study the role of CDCA5 in cell cycle progression and apoptosis. Moreover, subcutaneous xenograft tumour models were implemented to predict the efficacy of targeting CDCA5 in HCC in vivo. We found that CDCA5 expression was significantly higher in HCC tumour tissues, was associated with clinicopathological characteristics, and predicted poor overall survival in HCC patients. Silencing of CDCA5 with small interfering RNA (siRNA) inhibited cell proliferation and induced $\mathrm{G} 2 / \mathrm{M}$ cell cycle arrest in vitro. The xenograft growth assay revealed that CDCA5 downregulation
\end{abstract}

Correspondence to: Professor Kefeng Dou or Professor Kaishan Tao, Department of Hepatobiliary Surgery, Xijing Hospital, The Fourth Military Medical University, Xi'an, Shaanxi 710032, P.R. China

E-mail: doukef@fmmu.edu.cn

E-mail: taokaishan0686@163.com

"Contributed equally

Abbreviations: HCC, hepatocellular carcinoma; CDCA5, cell division cycle associated 5; ERK, extracellular signal-regulated kinase; qRT-PCR, quantitative real-time polymerase chain reaction; IHC, immunohistochemistry; p-AKTS, AKT (ser473); p-AKTT, AKT (Thr308); ANLTs, adjacent non-tumour liver tissues

Key words: CDCA5, proliferation, cell cycle, ERK/AKT pathway, hepatocellular carcinoma impeded HCC growth in vivo. Further study indicated that CDCA5 depletion decreased the levels of ERK1/2 and AKT phosphorylation in vitro and in vivo. Taken together, these results indicate that CDCA5 may act as a novel prognostic biomarker and therapeutic target for HCC.

\section{Introduction}

Hepatocellular carcinoma (HCC) is the sixth most aggressive human malignancy and the third leading cause of cancer-related mortality worldwide (1). In addition to genetic and epigenetic alterations, positive $\mathrm{HBV}$ and $\mathrm{HCV}$ infection, aflatoxin exposure, smoking, obesity and diabetes are considered the main risk factors associated with the incidence of $\operatorname{HCC}(2,3)$. Although some progress has been achieved in regards to clinical treatments, the overall survival (OS) of patients with HCC remains poor (4). Hence, a greater understanding of the molecular mechanisms underlying HCC tumourigenesis may provide new insights into functional pathogenic and therapeutic strategies.

Human cell division cycle associated 5 (CDCA5), also known as sororin and encoded by $C D C A 5$, was identified from a gene expression meta-analysis screen of cell cycle-associated (CDCA) transcripts $(5,6)$. CDCA5 is a key regulator of sister chromatid cohesion and separation, is involved in the generation and maintenance of sister chromatid cohesion and regulates the removal of cohesin from chromatin $(6,7)$. CDCA5 is required for sister chromatid cohesion during $\mathrm{S}$ and $\mathrm{G} 2$ phases, degraded through anaphase-promoting complex (APC)-dependent ubiquitination during G1 phase, and phosphorylated and dissociated from chromatids during prophase/prometaphase (8-10). In addition, CDCA5 was found to be significantly upregulated in human tumour tissues, including lung cancer, oral squamous cell carcinoma, urothelial cancers, gastric cancer and HCC (11-16), and silencing of CDCA5 in lung cancer and oral squamous cell carcinoma was found to result in decreased cell growth in vivo and in vitro $(11,14)$. However, knowledge of the biological and clinical significance of CDCA5 in HCC remains minimal, which prompted us to explore the functional roles of CDCA5 in HCC pathogenesis.

In the present study, we examined CDCA5 expression in clinical HCC samples and demonstrated that aberrant CDCA5 
expression in HCC tissues is correlated with multiple malignant clinicopathological characteristics and is an independent prognostic factor for the OS of individuals with HCC. Both in vitro and in vivo assays showed that silencing of CDCA5 inhibited HCC cell growth. The molecular mechanism underlying these effects involved inactivation of the ERK/AKT signaling pathway. These findings reveal that targeting CDCA5 is likely a promising strategy for HCC treatment.

\section{Materials and methods}

Cell lines and tissue samples. The HCC cell lines MHCC97-H and Huh7 were obtained from the Cell Bank of the Chinese Academy of Sciences (Shanghai, China). All cell lines were maintained in Dulbecco's Modified Eagle's medium (DMEM) supplemented with $10 \%$ fetal bovine serum (FBS), $100 \mathrm{U} / \mathrm{ml}$ penicillin and $100 \mu \mathrm{g} / \mathrm{ml}$ streptomycin and then incubated at $37^{\circ} \mathrm{C}$ in a humidified atmosphere containing $5 \% \mathrm{CO}_{2}$. Additionally, $30 \mathrm{HCC}$ samples and matched adjacent normal tissues from HCC patients were obtained at Xijing Hospital (Xi'an, China) between December 2014 and November 2015. The mean ages of these patients were 53.8 years, and the percentage of male was $76.7 \%$. The study protocol was approved by the Ethics Committee of Xijing Hospital, and all participants provided written informed consent.

Immunohistochemistry. A commercially available tissue microarray (TMA) was purchased from Shanghai Outdo Biotech Co., Ltd. (Shanghai, China). CDCA5 protein expression in 98 paired paraffin-embedded tumour tissues and adjacent normal tissues was detected using an immunoperoxidase method. In brief, after deparaffinization, antigen retrieval was performed in a sodium citrate solution ( $\mathrm{pH}$ 6.0). After the sections were blocked with normal goat serum, they were incubated with rabbit anti-CDCA5 (1:100; cat. no. ab192237; Abcam) overnight at $4^{\circ} \mathrm{C}$. Subsequently, the sections were washed and incubated with horseradish peroxidase (HRP)-conjugated anti-rabbit IgG at room temperature for $1 \mathrm{~h}$. Finally, 3,3'-diaminobenzidine tetrahydrochloride substrate (DAB; ZSGB-BIO, Beijing, China) was used as a substrate to visualize the bound antibody, and the sections were lightly counterstained with haematoxylin, dehydrated and mounted.

siRNA and plasmid transfection. The following siRNAs targeting CDCA5 were purchased from ShanghaGenePharma Co., Ltd., (Shanghai, China): Sense1, 5'-GAAAGCCCAUCG UCUUAAATT-3' and antisense1, 5'-UUUAAGACGAUG GGCUUUCTT-3'; sense2, 5'-GCCAGAGACUUGGAAAUG UTT-3' and antisense2, 5'-ACAUUUCCAAGUCUCUGG CTT-3'; sense3, 5'-GGCCAUGAAUGCCGAGUUUTT-3' and antisense 3, 5'-AAACUCGGCAUUCAUGGCCTT-3'. A negative control siRNA (si-NC) was also used: Sense, 5'-UUCUCCGAACGUGUCACGUTT-3' and antisense, 5'-ACGUGACAC GUUCGGAGAATT-3'. HCC cells were transfected with CDCA5 siRNA or si-NC using Invitrogen ${ }^{\mathrm{TM}}$ Lipofectamine 2000 (Thermo Fisher Scientific, Inc., Waltham, MA, USA) following the manufacturer's instructions. Lentiviral plasmids containing short hairpin RNA (shRNA) sequences either targeting human CDCA5 (shCDCA5) or serving as a negative control (shNC) were designed and produced by Shanghai GeneChem. MHCC97-H cells were plated in 6-well plates and incubated overnight. The culture medium was replaced with transduction-enhancing solution containing lentivirus at $20 \mathrm{MOI}$ and $50 \mu \mathrm{g} / \mathrm{ml}$ polybrene. After $12 \mathrm{~h}$, the medium was replaced with complete medium.

$R N A$ extraction and $q R T-P C R$. Total RNA was isolated using RNAiso Plus reagent (Takara Biotechnology Co., Ltd., Dalian, China) from the HCC cell lines or frozen tissue samples according to the manufacturer's protocol. Reverse transcription was conducted with PrimeScript ${ }^{\mathrm{TM}}$ Master Mix (Takara Biotechnology) at $37^{\circ} \mathrm{C}$ for $15 \mathrm{~min}$. Gene mRNA levels were determined using SYBR Premix EX Taq $^{\mathrm{TM}}$ II (Takara Biotechnology) on a Bio-Rad IQ ${ }^{\mathrm{TM}} 5$ detection system (Bio-Rad Laboratories, Inc., Hercules, CA, USA). The PCR conditions were as follows: Pre-denaturation at $95^{\circ} \mathrm{C}$ for $30 \mathrm{sec} ; 40$ cycles of denaturation $\left(95^{\circ} \mathrm{C}\right.$ for $\left.5 \mathrm{sec}\right)$, annealing $\left(60^{\circ} \mathrm{C}\right.$ for $\left.30 \mathrm{sec}\right) . \beta$-actin was used as a quantitative control to normalize the mRNA expression levels of the target genes. All experiments were performed according to the manufacturer's instructions. The data were analysed with the comparative $\mathrm{Ct}$ $\left(2^{-\Delta \Delta \mathrm{Cq})}\right.$ method. The primer was designed and synthesized by Shanghai Genechem Co., Ltd., for CDCA5 (forward primer, GACGCCAGAGACTTGGAAATG and reverse primer, GGA CCTCGGTGAGTTTGGAG); $\beta$-actin (forward primer, CAT GTACGTTGCTATCCAGGC and reverse primer, CTCCTT AATGTCACGCACGAT).

Western blotting. Protein samples were prepared according to normal procedures with fresh lysis buffer (Beyotime Institute of Biotechnology, Haimen, China) containing protease and phosphatase inhibitor cocktails (Sigma-Aldrich; Merck KGaA, Darmstadt, Germany). Total protein extracts were separated by $10 \%$ sodium dodecyl sulfate-polyacrylamide gel electrophoresis (SDS-PAGE), transferred to PVDF membranes (Millipore, Billerica, MA, USA), and sequentially incubated with primary antibodies targeting CDCA5 (1:1,000; cat. no. ab192237; Abcam), ERK1/2 (1:1,000; 4695; Cell Signaling Technology, Inc., Danvers, MA, USA), p-ERK1/2 (1:1,000; cat. no. 4370; Cell Signaling Technology, Inc.), AKT, p-AKT (Thr308), p-AKT (Ser473) (1:1,000; cat. no. 9916T; Cell Signaling Technology, Inc.) and $\beta$-actin (1:1,000; cat. no. 3700; Cell Signaling Technology, Inc.) overnight at $4{ }^{\circ} \mathrm{C}$. Afterward, the membranes were incubated with horseradish peroxidase (HRP)-conjugated secondary antibodies (1:3,000; ProteinTech Group, Wuhan, China) at room temperature for $1 \mathrm{~h}$. The blots were visualized and imaged on a Bio-Rad ChemiDoc ${ }^{\mathrm{TM}} \mathrm{XRS}+$, and the images were analysed using Image $\mathrm{Lab}^{\mathrm{TM}}$ software (Bio-Rad Laboratories).

Cell proliferation and colony formation assays. Transfected MHCC97-H and Huh7 cells were seeded in 96-well plates at 2,000 cells/well, and cell proliferation was measured at 1, 2, 3, 4 and 5 days after seeding using Cell Counting Kit-8 (CCK-8; Yiyuan Biotechnologies, Guangzhou, China) assay according to the manufacturer's instructions. The experiment was performed with three replicates. Colony formation assays were performed in 6-well culture plates with 200 cells/well. The cells were incubated for 2 weeks in DMEM supplemented with $10 \%$ FBS. The colonies were then washed twice with 


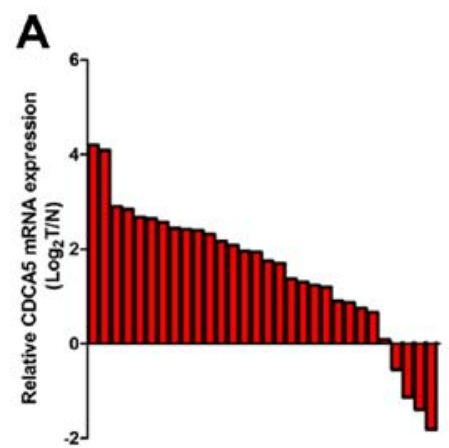

B

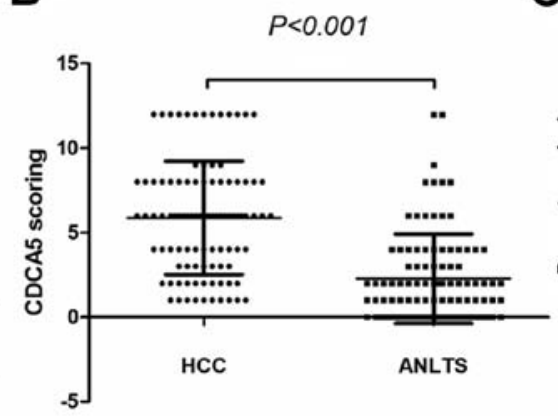

C

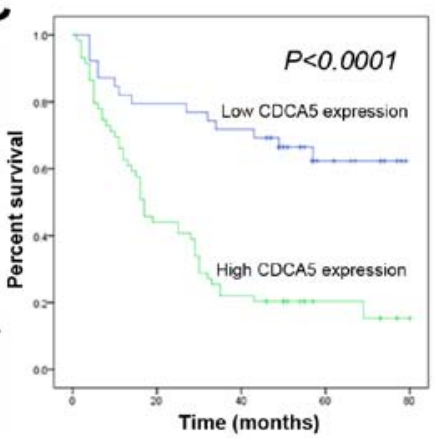

D

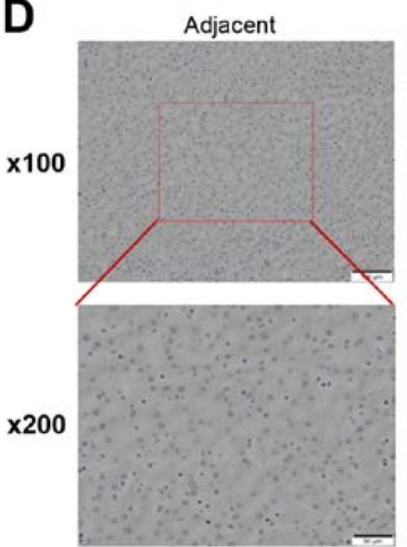

Carcinoma (I)

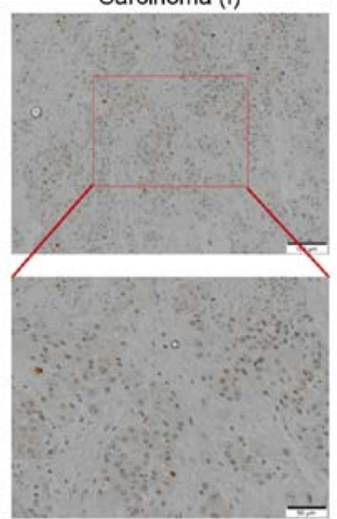

Carcinoma (II)

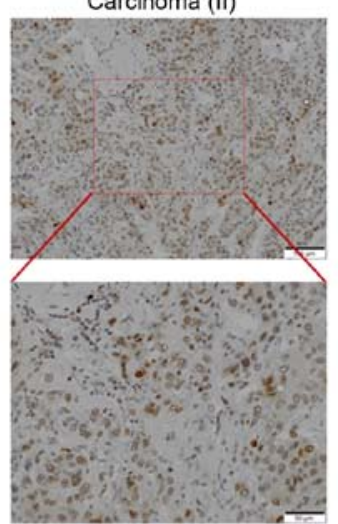

Carcinoma (III)

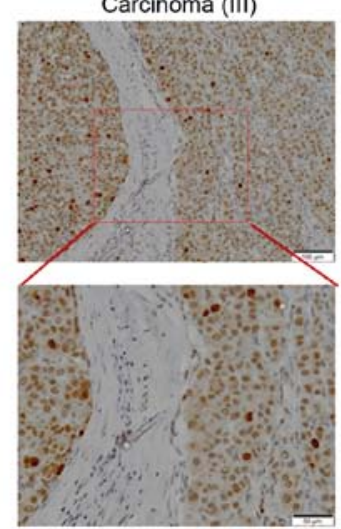

Figure 1. CDCA5 expression is frequently upregulated in HCC tissues. (A) CDCA5 mRNA expression levels in 30 pairs of HCC tissues and matched adjacent non-tumour tissues were evaluated by qRT-PCR. (B) Relative IHC staining for CDCA5 expression in HCC tissues and paired adjacent non-tumour liver tissues $(n=98)$. (C) CDCA5 expression was associated with overall survival in HCC patients. (D) Representative IHC image of HCC tissues and adjacent non-tumour liver tissues. HCC, hepatocellular carcinoma; IHC, immunohistochemistry; CDCA5, cell division cycle associated 5; ANLTs, adjacent non-tumour liver tissues.

phosphate-buffered saline (PBS), fixed with $95 \%$ ethanol and stained with a $4 \mathrm{~g} / \mathrm{l}$ crystal violet solution. The number of colonies containing over 50 cells was counted using a microscope (Leica Microsystems, Wetzlar, Germany).

Cell cycle and apoptosis analysis. Cells were transfected for $48 \mathrm{~h}$ with either si-CDCA5 or si-NC using Lipofectamine 2000. After transfection, the cells were collected and washed twice with ice-cold PBS. Next, the prepared cells were stained with $500 \mu \mathrm{l}$ of Cell Cycle Rapid Detection Solution (Dakewe, Beijing, China) and $50 \mathrm{~g} / \mathrm{ml}$ propidium iodide (PI) (Sigma-Aldrich; Merck KGaA; P4170) for $15 \mathrm{~min}$ and analysed using a Cytomics FC 500 flow cytometer (Beckman Coulter, Fullerton, CA, USA). Apoptosis was assayed by staining the cells with Annexin V-FITC and PI following the manufacturer's instructions and detecting the fluorescence using a flow cytometer.

Tumour growth in nude mice. Five male BALB/c nude mice at 4-week-old, weighing 15-18 g, were obtained from SLAC Laboratory Animal Co., Ltd. (Shanghai, China) and were bred in a special pathogen-free (SPF) grade laboratory in Fourth Military Medical University. The mice were housed 5-10/cage, in a 12-h light/dark cycle environment, with ad libitum access to food and water. Lentiviral transfected MHCC97-H cells $\left(2 \times 10^{6}\right)$ were suspended in $150 \mu \mathrm{l}$ of PBS and subcutaneously injected into both flanks of nude mice. After 3 weeks, the mice were euthanized via $\mathrm{CO}_{2}$ inhalation according to animal care guidelines, and the tumours were harvested and weighed. The resulting primary tumours were fixed, paraffin-embedded and sectioned for subsequent immunohistochemical staining. All experimental procedures on animals were approved by the Institutional Animal Care and Use Committee of The Fourth Military Medical University (Xi'an, Shaanxi, China).

Xenograft model and tumour therapy. To establish the subcutaneous model, MHCC97-H cells $\left(2 \times 10^{6}\right)$ were subcutaneously injected into the right flanks of nude mice. The female BALB/c nude mice at 4-week-old, weighing 15-18 g, were obtained from SLAC Laboratory Animal Co., Ltd. (Shanghai, China). The housing conditions were the same as previously described. Cholesterol-conjugated CDCA5 siRNA for in vivo RNA interference and its negative control were designed and synthesized by RiboBio Co., Ltd. (Guangzhou, China). The mice were randomly selected for treatment with CDCA5 siRNA or the negative control when the tumour diameter reached $5 \mathrm{~mm}(\mathrm{n}=3$ mice per group). Then, the tumours were injected with CDCA5 siRNA or the negative control every 3 days for 3 weeks.

Statistical analysis. Each experiment was repeated at least three times. All the data are generally expressed as the mean \pm SD. Differences between groups with homogenous variance were analysed using the Student's t-test. The $\chi^{2}$ test was used to analyse correlations between CDCA5 expression and the clinicopathological features. Survival curves were 
Table I. Correlation between CDCA5 expression and clinicopathological characteristics of the HCC cases.

\begin{tabular}{|c|c|c|c|c|}
\hline \multirow{2}{*}{$\begin{array}{l}\text { HCC clinical } \\
\text { variables }\end{array}$} & \multicolumn{2}{|c|}{ CDCA5 expression levels } & \multirow[b]{2}{*}{$\chi^{2}$} & \multirow[b]{2}{*}{ P-value } \\
\hline & Low $(n=39)$ & High $(n=59)$ & & \\
\hline Sex & & & 1.234 & 0.266 \\
\hline Male & 32 & 53 & & \\
\hline Female & 7 & 6 & & \\
\hline Age (years) & & & 1.448 & 0.229 \\
\hline$<50$ & 10 & 22 & & \\
\hline$\geq 50$ & 29 & 37 & & \\
\hline Cirrhosis & & & 1.486 & 0.223 \\
\hline Absence & 21 & 39 & & \\
\hline Presence & 18 & 20 & & \\
\hline Metastasis & & & 1.274 & $0.391^{\mathrm{a}}$ \\
\hline Absence & 35 & 48 & & \\
\hline Presence & 4 & 11 & & \\
\hline Histological grade & & & 4.506 & $0.034^{\mathrm{b}}$ \\
\hline I-II & 30 & 33 & & \\
\hline III & 9 & 26 & & \\
\hline Maximal tumor size $(\mathrm{cm})$ & & & 7.090 & $0.003^{\mathrm{b}}$ \\
\hline$<5$ & 23 & 17 & & \\
\hline$\geq 5$ & 16 & 42 & & \\
\hline Tumor number & & & 2.266 & 0.132 \\
\hline Single & 29 & 37 & & \\
\hline Multiple & 8 & 21 & & \\
\hline TNM stage & & & 5.320 & $0.019^{\mathrm{b}}$ \\
\hline I-II & 24 & 22 & & \\
\hline III-IV & 15 & 37 & & \\
\hline
\end{tabular}

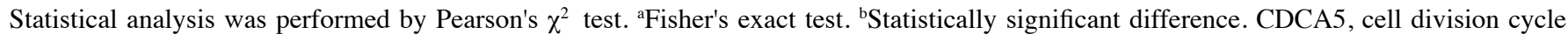
associated 5; HCC, hepatocellular carcinoma; TNM, tumor, node, metastasis.

calculated using the Kaplan-Meier method and compared by log-rank test. Cox proportional hazards regression analysis was used to identify risk factors. Statistical analyses were performed using SPSS 22.0 for Windows (IBM Corp., Armonk, NY, USA). A probability value $(\mathrm{P})<0.05$ was indicative of statistical significance.

\section{Results}

CDCA5 is frequently upregulated in HCC tissues and is strongly associated with clinicopathological characteristics and predicts a poor prognosis in HCC patients. To compare the CDCA5 expression pattern in HCC tumour tissues and adjacent non-tumour liver tissues (ANLTs), we first detected CDCA5 mRNA expression in 30 pairs of matched HCC and non-tumour tissue samples by quantitative real-time PCR. We found that the mRNA expression levels of CDCA5 were significantly elevated in the HCC tissues of most patients (86.67\%) (Fig. 1A). Then, we used immunohistochemistry (IHC) to investigate the CDCA5 protein expression in 98 pairs of matched HCC samples and ANLTs. In agreement with the qRT-PCR results, the protein levels of CDCA5 were significantly higher in HCC tissues from most of the patients (80 of 98) (Fig. 1B). Representative IHC staining results are shown in Fig. 1D, which indicated that CDCA5 expression was enriched in the nucleus. Furthermore, the CDCA5 immunoreactivity scores showed that CDCA5 protein expression was significantly correlated with histological grade $(\mathrm{P}=0.034)$, tumour size $(\mathrm{P}=0.003)$ and $\mathrm{TNM}$ stage $(\mathrm{P}=0.019)$. No significant association was found between CDCA5 expression and the other clinicopathological characteristics, including sex $(\mathrm{P}=0.266)$, age $(\mathrm{P}=0.229)$, cirrhosis $(\mathrm{P}=0.223)$, metastasis $(\mathrm{P}=0.391)$ and tumour number $(\mathrm{P}=0.132)$ (Table $\mathrm{I})$.

The Kaplan-Meier survival analysis revealed that patients with elevated CDCA5 expression had a shorter OS than those with low levels of CDCA5 expression (Fig. 1C, $\mathrm{P}<0.0001$ ). The multivariate Cox regression analysis assessed whether elevated CDCA5 expression in tumour cells was an independent prognostic factor for HCC. CDCA5 expression ( $\mathrm{P}=0.001)$ and TNM stage $(\mathrm{P}=0.005)$ were independent prognostic factors for survival in HCC patients (Table II). Taken together, these results revealed that CDCA5 plays an oncogenic role in 
A

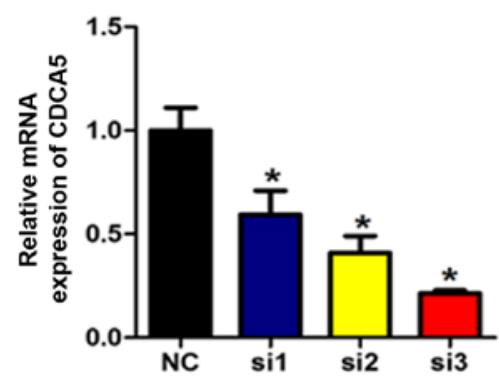

C

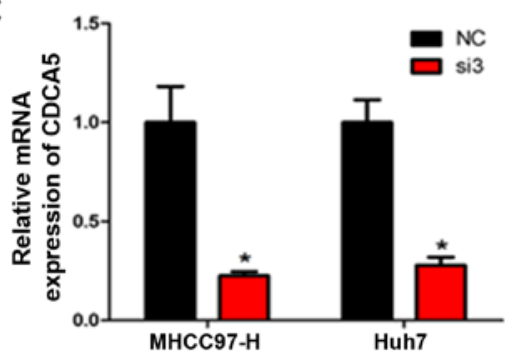

D

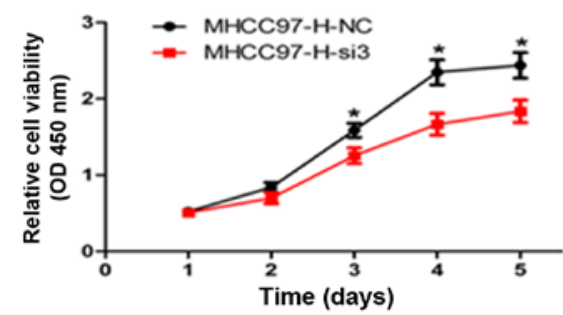

$\mathbf{E}$

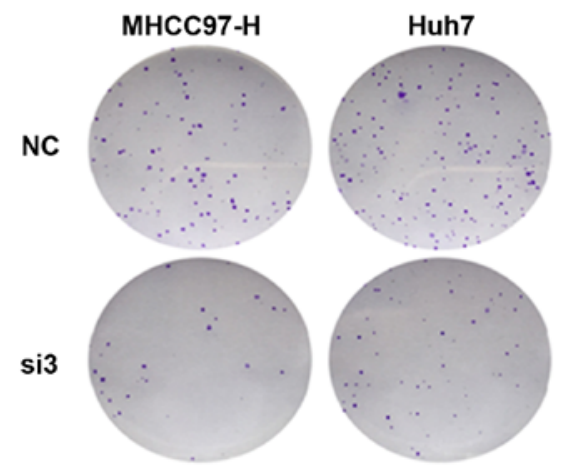

B
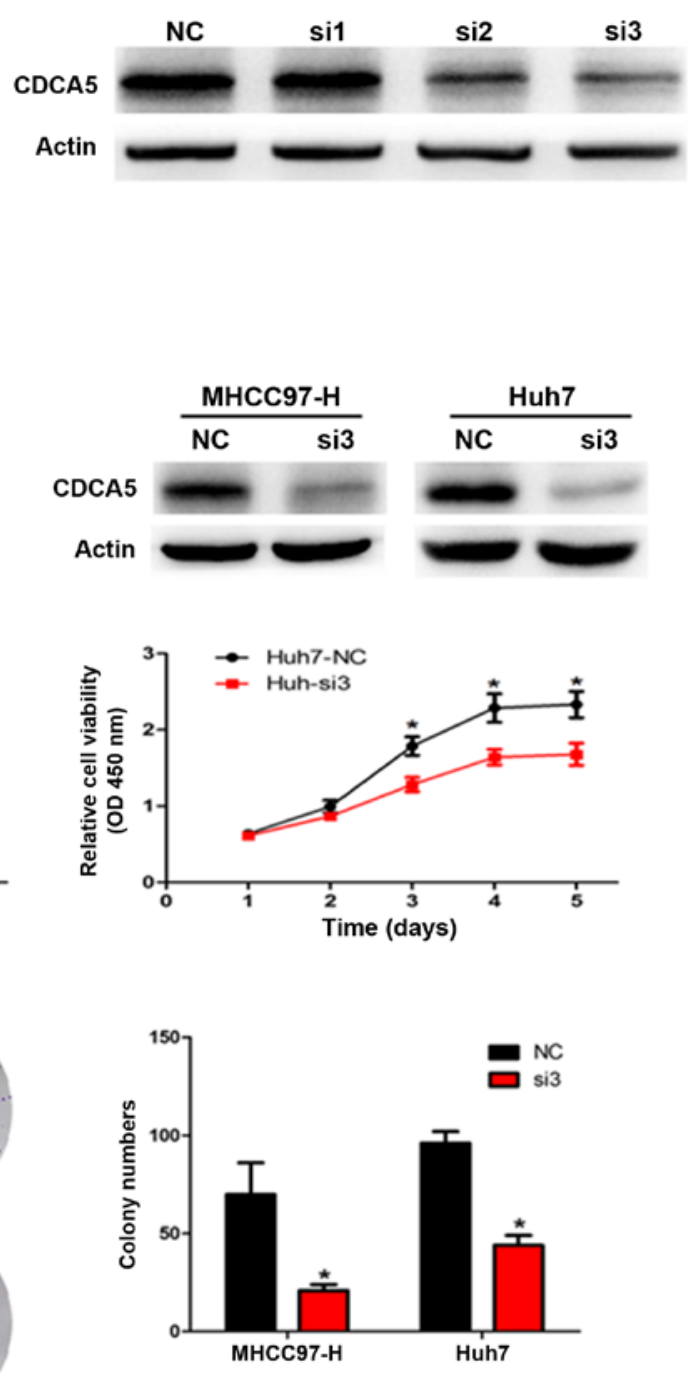

Figure 2. Silencing of CDCA5 inhibits HCC cell proliferation and clonogenicity. (A and B) Quantitation of CDCA5 mRNA and protein levels in MHCC97-H cells by qRT-PCR and western blotting after transfection with different siRNAs targeting CDCA5 (si1, si2 and si3) or a negative control siRNA (NC). (C) CDCA5 expression in MHCC97-H and Huh7 cells after transfection with si3 and NC. (D) The proliferation curves of MHCC97-H and Huh7 cells after CDCA5 knockdown. (E) A colony formation assay was used to measure the clonogenicity of MHCC97-H and Huh7 cells after CDCA5 knockdown. "P<0.05. CDCA5, cell division cycle associated 5; HCC, hepatocellular carcinoma.

HCC and may be used as an independent factor for predicting the prognosis of HCC patients.

Silencing of CDCA5 inhibits HCC cell proliferation and clonogenicity in vitro. Given that CDCA5 expression levels were positively associated with several progressive clinicopathological features, including tumour size, we transfected MHCC97-H and Huh7 cells with siRNA targeting CDCA5 to knock down endogenous CDCA5 expression and examined the proliferation in these cells. qRT-PCR and western blot analyses were performed to verify the reduction in CDCA5 levels (Fig. 2A-C). All three CDCA5 siRNAs decreased CDCA5 expression, with the most effective siRNA (si-3) used for all subsequent experiments. The CCK- 8 assay showed that CDCA5 downregulation markedly reduced cell proliferation in the MHCC97-H and Huh7 cells (Fig. 2D). To evaluate the long-term effects of CDCA5 on cell proliferation, the colony formation assay was performed. Our results showed that CDCA5 knockdown significantly decreased the number of colonies (Fig. 2E). Taken together, these results indicate that silencing of CDCA5 inhibited HCC cell proliferation and clonogenicity in vitro.

Silencing of CDCA5 induces cell cycle arrest in HCC. To further investigate the mechanisms by which reduced CDCA5 expression suppresses HCC cell growth, we measured the DNA content using flow cytometry in HCC cells transfected with siCDCA5. As shown in Fig. 3A, silencing of CDCA5 in MHCC97-H and Huh7 cells led to an increase in the percentage of cells in the G2/M phase. However, there was no 
Table II. Univariate and multivariate analyses of factors associated with the overall survival of the HCC patients.

Variables

$\operatorname{HR}(95 \% \mathrm{CI})$

P-value

Univariate analysis (Cox: Enter)

Sex (male/female)

Age $(<50 / \geq 50$ years $)$

$1.712(0.737-3.975)$

0.211

Liver cirrhosis (absence/presence)

$0.834(0.492-1.411)$

0.498

Metastasis (absence/presence)

$1.045(0.626-1.744)$

0.867

Histological grade (I-II/III)

$2.993(1.634-5.483)$

$<0.001^{\mathrm{a}}$

Tumor size $(<5 / \geq 5 \mathrm{~cm})$

$0.839(0.650-1.082)$

0.175

$2.744(1.544-4.844)$

$<0.001^{\mathrm{a}}$

Tumor number (single/multiple)

$1.467(0.828-2.599)$

0.189

TNM stage (I-II/III- IV)

$2.944(1.707-5.075)$

CDCA5 (low/high)

$3.632(1.984-6.647)$

$<0.001^{\mathrm{a}}$

$<0.001^{\mathrm{a}}$

Multivariate analysis (Cox: Forward LR)

TNM stage (I-II/III- IV)

A
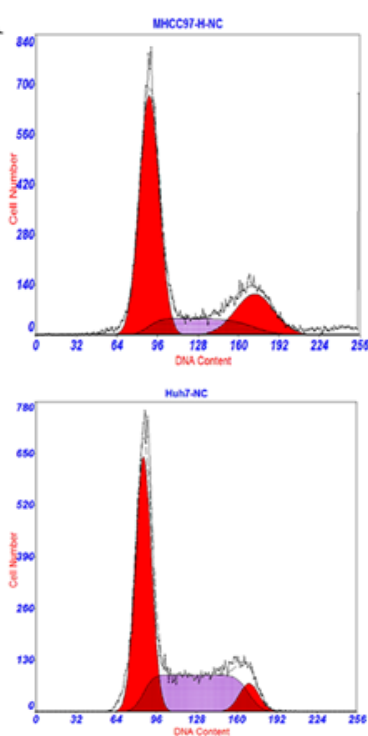

B
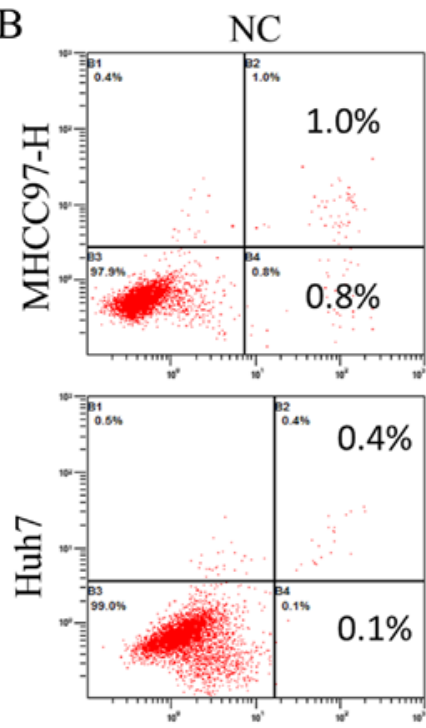
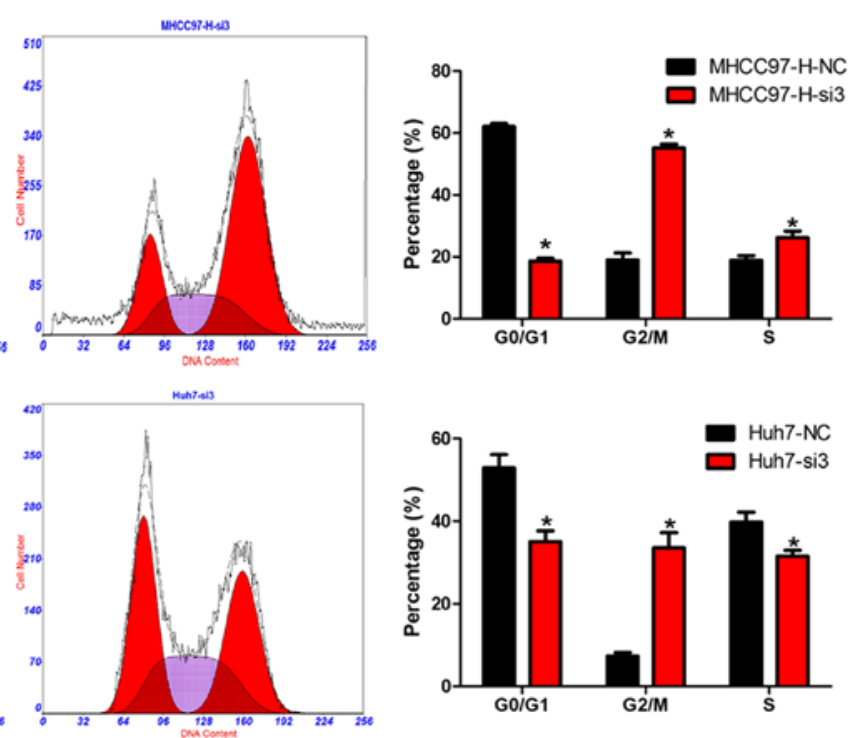

si3
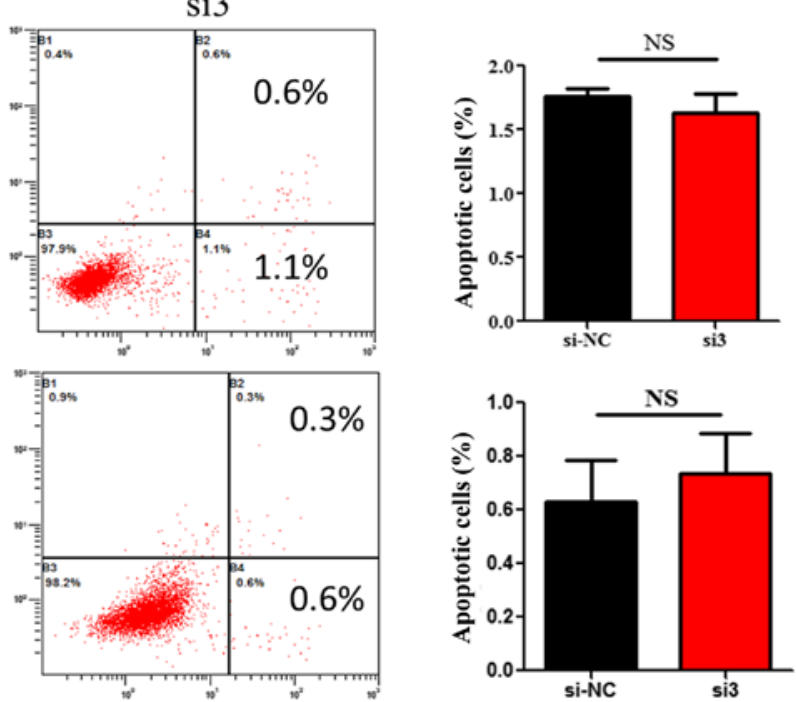

Figure 3. Silencing of CDCA5 induces HCC cell cycle arrest. (A) Cell cycle analysis of MHCC97-H and Huh7 cell lines after CDCA5 knockdown. (B) Analysis of apoptosis of MHCC97-H and Huh7 cell lines after CDCA5 knockdown. "P<0.05; NS, not significant; CDCA5, cell division cycle associated 5; HCC, hepatocellular carcinoma. 
A

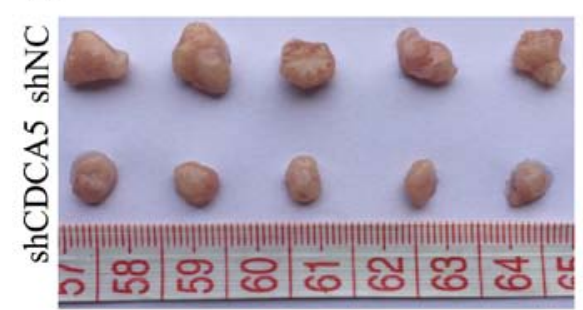

B

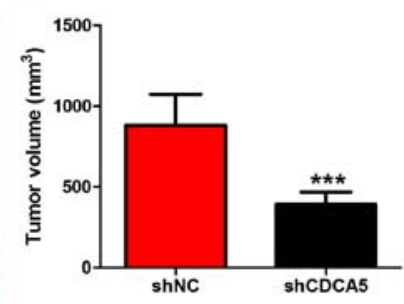

C

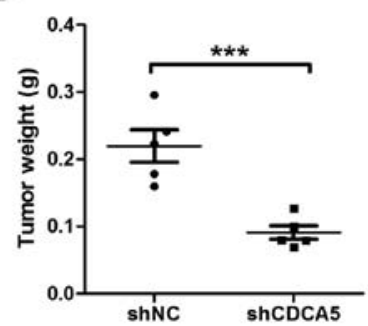

D
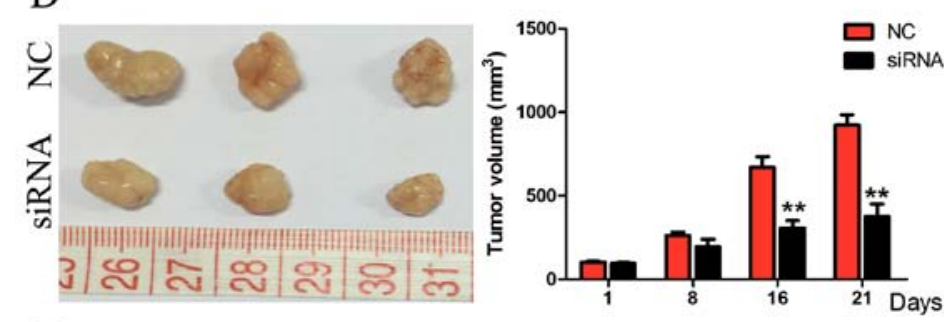

E

CDCA5
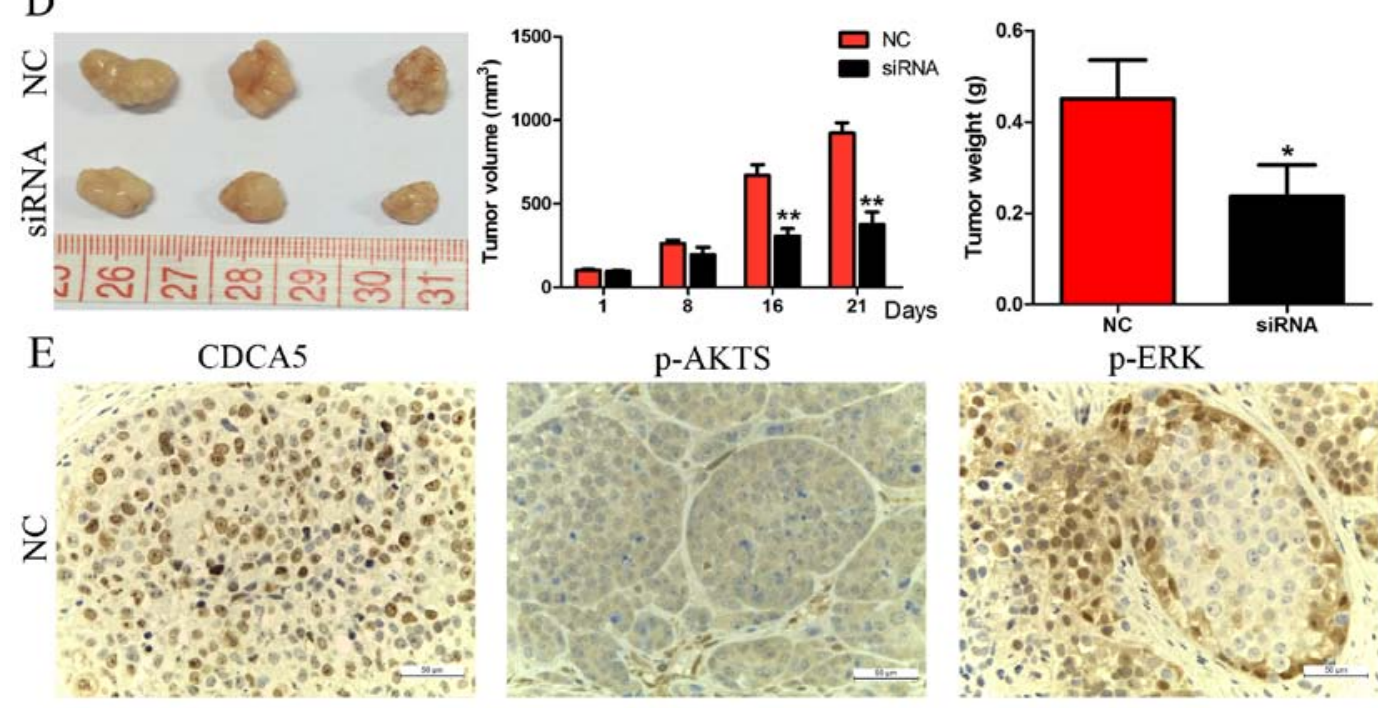

p-ERK
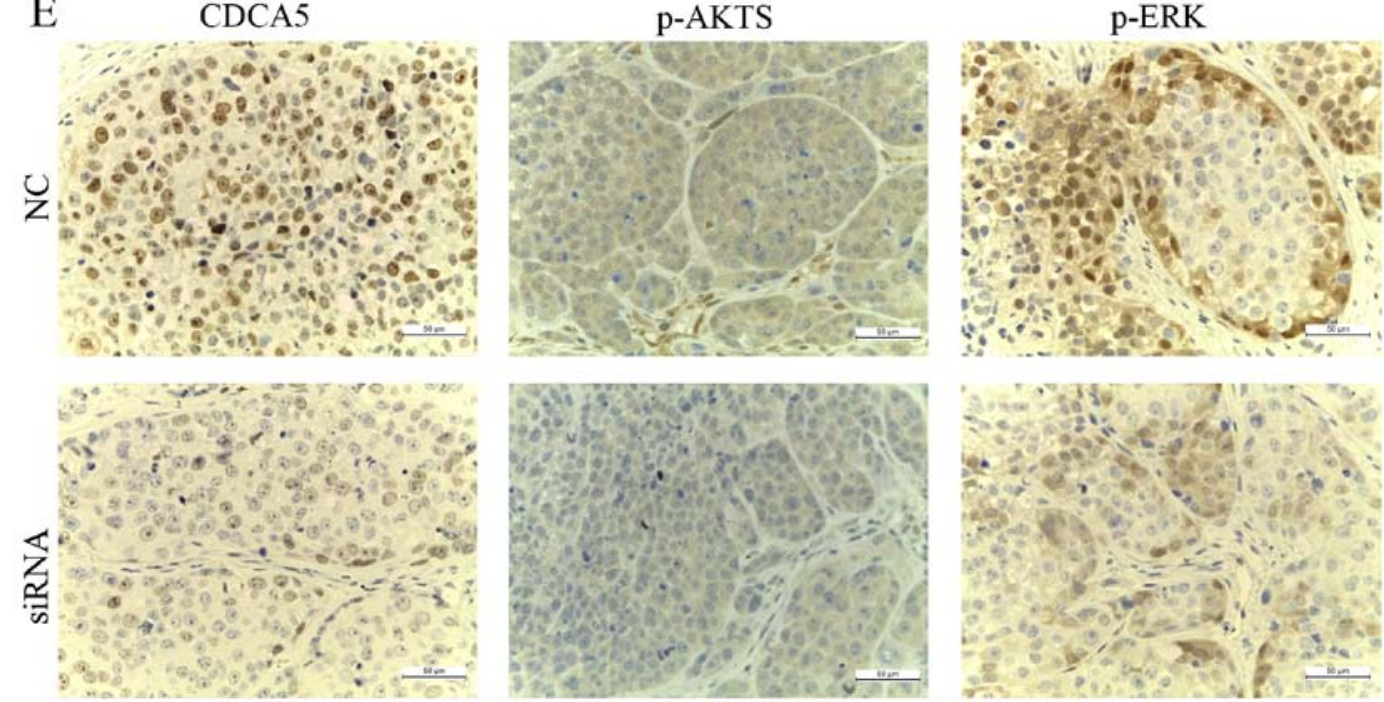

Figure 4. CDCA5 inhibition attenuates tumour growth in subcutaneous HCC models. (A and B) Tumours were collected from the sacrificed mice, and the size of tumours was compared. (C) Tumour weights were measured after mice were sacrificed and are shown as the means \pm SEM. (D) Cholesterol-conjugated CDCA5 siRNA significantly decreased tumour size and weight compared with the negative control (NC). (E) IHC analysis of CDCA5, p-AKTS and p-ERK expression in xenograft tumours which were injected with cholesterol-conjugated CDCA5 siRNA and negative control (NC) (magnification, $\mathrm{x} 400$ ). ${ }^{*} \mathrm{P}<0.05$, ${ }^{* *} \mathrm{P}<0.01,{ }^{* * *} \mathrm{P}<0.001$. CDCA5, cell division cycle associated 5; HCC, hepatocellular carcinoma; IHC, immunohistochemistry; p-AKTS, AKT (ser473).

significant difference in the percentage of HCC cells in terms of apoptosis between the CDCA5-siRNA group and the si-NC group (Fig. 3B). These results suggest that the suppressive effect of silencing CDCA5 on HCC cell growth may only be attributed to cell cycle arrest.

Targeting CDCA5 inhibits HCC tumour growth in vivo. The effect of CDCA5 knockdown on the growth of HCC cells was further confirmed by tumour growth assays in nude mice. MHCC97-H cells transfected with either shNC or shCDCA5 were injected into nude mice for xenotransplantation. The results of this experiment showed that CDCA5 knockdown significantly inhibited tumour growth. As shown in Fig. 4A and B, mice injected with MHCC97-H-shCDCA5 cells showed significantly decreased tumour growth compared with mice injected with MHCC97-H-shNC cells. The results of tumour weight indicated that CDCA5 knockdown significantly decreased overall tumour weight (Fig. 4C, P<0.001). We further investigated the effect of therapeutic CDCA5 siRNA on $\mathrm{HCC}$ growth in vivo. After repeated injection (every 3 days for 3 weeks) of cholesterol-conjugated CDCA5 siRNA, we found that tumour growth was significantly decreased, as evidenced by the strongly reduced tumour size and weight compared with the effects of the control treatment (Fig. 4D), and CDCA5 protein expression was confirmed to be downregulated after treatment with CDCA5 siRNA (Fig. 4E). These data indicated that CDCA5 affects the growth of HCC cells in vivo.

Silencing of CDCA5 suppresses activation of the ERK and $A K T$ pathways. We next assessed the possible oncogenic pathways that are affected by changes in CDCA5 expression. Using western blot analysis, we detected a marked decrease in CDCA5 expression in MHCC97-H-si3 and Huh7-si3 cells, and the levels of phosphorylated ERK1/2 and AKT were downregulated in these cells (Fig. 5). IHC showed that the levels of phosphorylated ERK1/2 and AKT were also significantly 


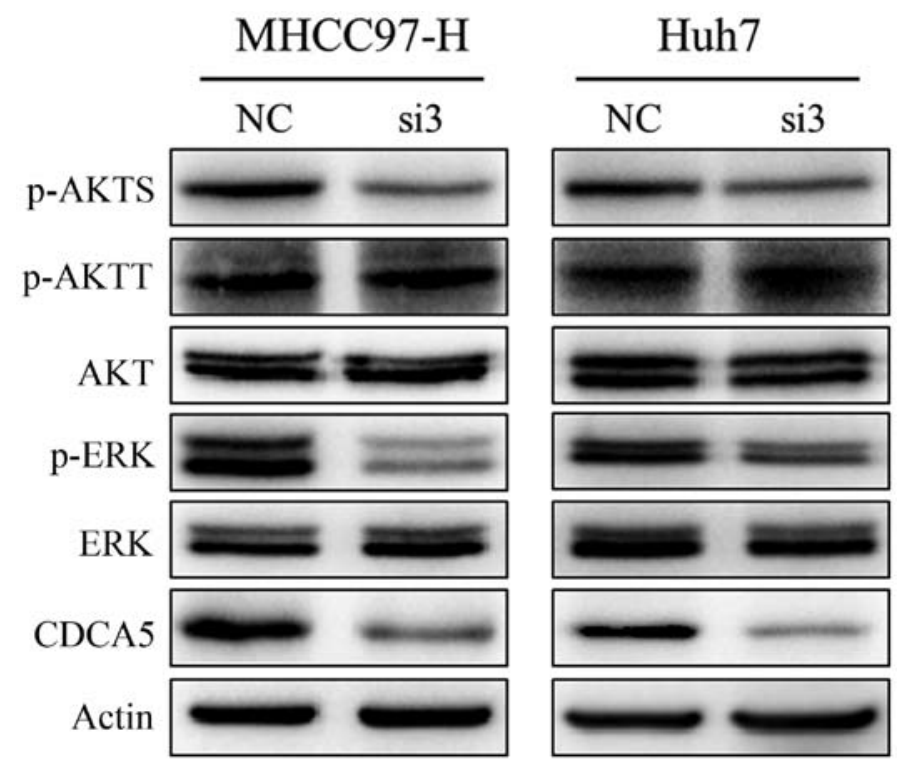

Figure 5. Silencing of CDCA5 suppresses activation of the ERK and AKT signaling pathways. Western blotting of CDCA5, ERK, p-ERK, AKT, p-AKTS and p-AKTT expression in cells transfected with si3 or NC. $\beta$-actin served as the loading control. CDCA5, cell division cycle associated 5; p-AKTS, AKT (ser473); p-AKTT, AKT (Thr308).

decreased in subcutaneous tumours of the CDCA5 siRNA treatment group (Fig. 4E). These results suggest that activation of the ERK and AKT pathways is responsible for CDCA5 regulation of HCC cell proliferation.

\section{Discussion}

Despite prominent advances in the detection and treatment of hepatocellular carcinoma (HCC), this disease is still the most common primary malignant tumour of the liver and has a low patient survival rate. Detecting prognostic molecular biomarkers is essential to help predict patient outcomes and select optimal therapeutic approaches against HCC (17). Currently, many studies have been devoted to exploring and identifying potential molecular biomarkers for HCC (18-21), but the molecular mechanisms underlying HCC are poorly understood. In addition, it is difficult to characterize individuals with HCC using only one biomarker as HCC develops and progresses with multiple risk factors, including the accumulation of genetic and epigenetic alterations (22). Therefore, we need to explore and identify more novel prognostic biomarkers and effective therapeutic targets to improve the survival of individuals with HCC. Recently, a study showed that CDCA5 mRNA expression was upregulated in HCC tissues, and predicted poor survival in a TCGA cohort (16). Similarly, in this study, we found that CDCA5 mRNA and protein expression was significantly upregulated in clinically collected HCC tissues. Overexpression of CDCA5 protein is strongly associated with histological grade, tumour size, and TNM stage, and predicts a poor prognosis in HCC patients. These data suggest that CDCA5 may be a useful prognostic molecular biomarker and therapeutic target for HCC.

CDCA5 was reported to be linked with cancer growth in vitro and/or in vivo $(11,14,23)$, and other cell division cycle-associated genes such as CDCA1 $(24,25)$, CDCA2 (26) and CDCA3 (27) have also been associated with cancer growth. Therefore, we performed in vitro cell proliferation and colony formation assays as well as in vivo xenograft growth assays to study the role of CDCA5 in HCC progression. Herein, our results suggested that CDCA5 knockdown can significantly inhibit HCC growth in vivo and in vitro. Cholesterol-conjugated siRNA delivery has been reported to be effective in gene silencing following either local injection or intravenous systemic injection $(28,29)$. In this study, cholesterol-conjugated CDCA5 siRNA was directly injected into the tumour mass. Unsurprisingly, after multiple injections of cholesterol-conjugated CDCA5 siRNA, the expression of CDCA5 was downregulated in the CDCA5 siRNA-treated tumours. Moreover, tumour growth was significantly decreased. Taken together, our data indicate that targeting CDCA5 may be a potentially useful therapeutic approach for HCC.

Alterations in cell growth capability are usually caused by abnormal cell cycle progression or apoptosis. Emerging evidence suggests that CDCA5 plays an important role in controlling cell cycle progression $(6,8,14)$. Therefore, we analysed the effects of CDCA5 on the cell cycle using flow cytometry. We found that silencing of CDCA5 induced cell cycle arrest of HCC cells in the G2/M phase. We also detected alterations in apoptosis after silencing of CDCA5 in HCC cells, but these changes were not significant between the si-CDCA5 group and the si-NC group. These data were inconsistent with recent research (16), perhaps because we used different method to knockdown CDCA5. Thus, further study is needed to explore its potential mechanisms. However, these results still demonstrated that CDCA5 plays an important role in HCC proliferation by controlling cell cycle progression. We also determined the inhibitory effect of CDCA5 on HCC cell migration and invasion ability by using Transwell assays. There was no significant difference between the si-CDCA5 group and si-NC group (data not shown). Overall, CDCA5 may only influence $\mathrm{HCC}$ malignant progression by regulating cell proliferation and cell cycle. 
To date, the mechanisms for CDCA5 function in hepatocarcinogenesis and HCC progression have been minimally elucidated. The ERK pathway is involved in regulating a wide variety of cellular processes, including proliferation, differentiation, transcription regulation and development (30-32). An increase in ERK expression and activity was found in HCC tissues $(33,34)$ and was closely related to hepatic carcinogenesis and progression (32). In this study, we found that silencing of CDCA5 decreased the levels of phosphorylated ERK in vitro and in vivo. Previous studies have demonstrated that activation of the PI3K/AKT pathway is also indispensable to $\mathrm{HCC}$ development and progression and can regulate the malignant behavior of HCC $(35,36)$. P-AKTT (Thr308) and P-AKTS (Ser473) are different phosphorylation sites of AKT. T308 and S473 phosphorylation occurs in response to growth factors and other extracellular stimuli, and is essential to maximal Akt activation (37). Furthermore, p-AKTS (ser473) is highly expressed in HCC tissues, and acts as a risk factor for early disease recurrence and poor prognosis in $\operatorname{HCC}(38,39)$. Herein, we found that silencing of CDCA5 significantly suppressed the expression of p-AKTS in vitro and in vivo. Taken together, these results indicate a possible mechanism by which silencing of CDCA5 inhibits HCC development and progression through regulation of the ERK/AKT pathway. Of course, further studies are necessary to explore the role of other molecular partners in CDCA5-mediated hepatocarcinogenesis.

In conclusion, we demonstrated that CDCA5 is highly expressed in HCC tumour tissues, and its increased expression is significantly associated with poor outcomes. In vitro and in vivo studies indicated that silencing of CDCA5 induces G2/M phase arrest and inhibits HCC cell growth. In addition, the treatment effect of targeting CDCA5 in HCC may be attributed to inactivation of the ERK/AKT pathway. Taken together, our results suggest that CDCA5 could serve as an indicator of poor prognosis and/or a therapeutic target in HCC.

\section{Acknowledgements}

We are grateful to Wei Liu and Dr Lin Wang for providing technical support.

\section{Funding}

The present study was supported by the National Natural Science Foundation of China (grant no. 81702329).

\section{Availability of data and materials}

The datasets used during the present study are available from the corresponding author upon reasonable request.

\section{Authors' contributions}

JW, CX, MP, KT and KD participated in the design of the study and drafting of the manuscript. JW and CX carried out most of the experiments. BD, XY, ZY, RS and RZ participated in the immunoassays and statistical analysis of the data. All authors approved the final manuscript and agree to be accountable for all aspects of the research in ensuring that the accuracy or integrity of any part of the work are appropriately investigated and resolved.

\section{Ethics approval and consent to participate}

The study protocol was approved by the Ethics Committee of Xijing Hospital, and all participants provided written informed consent. All experimental procedures on animals were approved by the Institutional Animal Care and Use Committee of Fourth Military Medical University.

\section{Patient consent for publication}

Not applicable.

\section{Competing interests}

The authors declare that they have no competing interests.

\section{References}

1. Forner A, Llovet JM and Bruix J: Hepatocellular carcinoma. Lancet 379: 1245-1255, 2012.

2. Wahid B, Ali A, Rafique S and Idrees M: New insights into the epigenetics of hepatocellular carcinoma. Biomed Res Int 2017: 1609575,2017

3. Herceg Z and Paliwal A: Epigenetic mechanisms in hepatocellular carcinoma: How environmental factors influence the epigenome. Mutat Res 727: 55-61, 2011.

4. Villanueva A, Hoshida Y, Battiston C, Tovar V, Sia D, Alsinet C, Cornella H, Liberzon A, Kobayashi M, Kumada H, et al: Combining clinical, pathology, and gene expression data to predict recurrence of hepatocellular carcinoma. Gastroenterology 140: 1501-1512.e2, 2011.

5. Walker MG: Drug target discovery by gene expression analysis: Cell cycle genes. Curr Cancer Drug Targets 1: 73-83, 2001.

6. Zhang $\mathrm{N}$ and Pati D: Sororin is a master regulator of sister chromatid cohesion and separation. Cell Cycle 11: 2073-2083, 2012.

7. Zhang $\mathrm{N}$ and Pati D: C-terminus of Sororin interacts with SA2 and regulates sister chromatid cohesion. Cell Cycle 14: 820-826, 2015.

8. Rankin S, Ayad NG and Kirschner MW: Sororin, a substrate of the anaphase-promoting complex, is required for sister chromatid cohesion in vertebrates. Mol Cell 18: 185-200, 2005.

9. Nishiyama T, Sykora MM, Huis in 't Veld PJ, Mechtler K and Peters JM: Aurora B and Cdk1 mediate Wapl activation and release of acetylated cohesin from chromosomes by phosphorylating Sororin. Proc Natl Acad Sci USA 110: 13404-13409, 2013.

10. Nishiyama T, Ladurner R, Schmitz J, Kreidl E, Schleiffer A, Bhaskara V, Bando M, Shirahige K, Hyman AA, Mechtler K, et al: Sororin mediates sister chromatid cohesion by antagonizing Wapl. Cell 143: 737-749, 2010.

11. Nguyen MH, Koinuma J, Ueda K, Ito T, Tsuchiya E, Nakamura Y and Daigo Y: Phosphorylation and activation of cell division cycle associated 5 by mitogen-activated protein kinase play a crucial role in human lung carcinogenesis. Cancer Res 70: 5337-5347, 2010.

12. Showe MK, Kossenkov AV and Showe LC: The peripheral immune response and lung cancer prognosis. Oncoimmunology 1: 1414-1416, 2012.

13. Chang IW, Lin VC, He HL, Hsu CT, Li CC, Wu WJ, Huang CN, Wu TF and Li CF: CDCA5 overexpression is an indicator of poor prognosis in patients with urothelial carcinomas of the upper urinary tract and urinary bladder. Am J Transl Res 7: 710-722, 2015.

14. Tokuzen N, Nakashiro K, Tanaka H,Iwamoto K and Hamakawa $H$ : Therapeutic potential of targeting cell division cycle associated 5 for oral squamous cell carcinoma. Oncotarget 7: 2343-2353, 2016.

15. Zhang Z, Shen M and Zhou G: Upregulation of CDCA5 promotes gastric cancer malignant progression via influencing cyclin E1. Biochem Biophys Res Commun 496: 482-489, 2018. 
16. Shen Z, Yu X, Zheng Y, Lai X, Li J, Hong Y, Zhang H, Chen C, Su Z and Guo R: CDCA5 regulates proliferation in hepatocellular carcinoma and has potential as a negative prognostic marker. Onco Targets Ther 11: 891-901, 2018.

17. Sia D, Villanueva A, Friedman SL and Llovet JM: Liver cancer cell of origin, molecular class, and effects on patient prognosis. Gastroenterology 152: 745-761, 2017.

18. Dhayat SA, Hüsing A, Senninger N, Schmidt HH, Haier J, Wolters H and Kabar I: Circulating microRNA-200 family as diagnostic marker in hepatocellular carcinoma. PLoS One 10: e140066, 2015.

19. Bagi CM and Andresen CJ: Models of hepatocellular carcinoma and biomarker strategy. Cancers 2: 1441-1452, 2010.

20. Wang W, Jia WD, Hu B and Pan YY: RAB10 overexpression promotes tumor growth and indicates poor prognosis of hepatocellular carcinoma. Oncotarget 8: 26434-26447, 2017.

21. Klingenberg M, Matsuda A,Diederichs S and Patel T: Non-coding RNA in hepatocellular carcinoma: Mechanisms, biomarkers and therapeutic targets. J Hepatol 67: 603-618, 2017.

22. Khan FS, Ali I, Afridi UK, Ishtiaq M and Mehmood R: Epigenetic mechanisms regulating the development of hepatocellular carcinoma and their promise for therapeutics. Hepatol Int 11: 45-53, 2017.

23. Kato T, Lee D, Wu L, Patel P, Young AJ, Wada H, Hu HP, Ujiie H, Kaji M, Kano S, et al: SORORIN and PLK1 as potential therapeutic targets in malignant pleural mesothelioma. Int J Oncol 49: 2411-2420, 2016

24. Tokuzumi A, Fukushima S, Miyashita A, Nakahara S, Kubo Y, Yamashita J, Harada M, Nakamura K, Kajihara I, Jinnin M and Ihn H: Cell division cycle-associated protein 1 as a new melanoma-associated antigen. J Dermatol 43: 1399-1405, 2016.

25. Kobayashi Y, Takano A, Miyagi Y, Tsuchiya E, Sonoda H, Shimizu T, Okabe H, Tani T, Fujiyama Y and Daigo Y: Cell division cycle-associated protein 1 overexpression is essential for the malignant potential of colorectal cancers. Int J Oncol 44: 69-77, 2014.

26. Shi R, Zhang C, Wu Y, Wang X, Sun Q, Sun J, Xia W, Dong G, Wang A, Jiang $\mathrm{F}$ and $\mathrm{Xu} \mathrm{L}$ : CDCA2 promotes lung adenocarcinoma cell proliferation and predicts poor survival in lung adenocarcinoma patients. Oncotarget 8: 19768-19779, 2017.

27. Adams MN, Burgess JT, He Y, Gately K, Snell C, Zhang SD, Hooper JD, Richard DJ and O'Byrne KJ: Expression of CDCA3 is a prognostic biomarker and potential therapeutic target in non-small cell lung cancer. J Thorac Oncol 12: 1071-1084, 2017.

28. Hou J, Lin L, Zhou W, Wang Z, Ding G, Dong Q, Qin L, Wu X, Zheng Y, Yang Y, et al: Identification of miRNomes in human liver and hepatocellular carcinoma reveals miR-199a/b-3p as therapeutic target for hepatocellular carcinoma. Cancer Cell 19: 232-243, 2011
29. Hou J, Zhou Y, Zheng Y, Fan J, Zhou W, Ng IO, Sun H, Qin L, Qiu S, Lee JM, et al: Hepatic RIG-I predicts survival and interferon-alpha therapeutic response in hepatocellular carcinoma. Cancer Cell 25: 49-63, 2014.

30. Burotto M, Chiou VL, Lee JM and Kohn EC: The MAPK pathway across different malignancies: A new perspective. Cancer 120: 3446-3456, 2014.

31. Chang L and Karin M: Mammalian MAP kinase signalling cascades. Nature 410: 37-40, 2001.

32. Wang S, Huang X, Li Y, Lao H, Zhang Y, Dong H, Xu W, Li JL and Li M: RN181 suppresses hepatocellular carcinoma growth by inhibition of the ERK/MAPK pathway. Hepatology 53: 1932-1942, 2011

33. Schmidt CM, McKillop IH, Cahill PA and Sitzmann JV: Increased MAPK expression and activity in primary human hepatocellular carcinoma. Biochem Biophys Res Commun 236: 54-58, 1997.

34. Tsuboi Y, Ichida T, Sugitani S, Genda T, Inayoshi J, Takamura M, Matsuda Y, Nomoto M and Aoyagi Y: Overexpression of extracellular signal-regulated protein kinase and its correlation with proliferation in human hepatocellular carcinoma. Liver Int 24: 432-436, 2004

35. Courtney KD, Corcoran RB and Engelman JA: The PI3K pathway as drug target in human cancer. J Clin Oncol 28: 1075-1083, 2010.

36. Fresno Vara JA, Casado E, de Castro J, Cejas P, Belda-Iniesta C and González-Barón M: PI3K/Akt signalling pathway and cancer. Cancer Treat Rev 30: 193-204, 2004.

37. Alessi DR, Andjelkovic M, Caudwell B, Cron P, Morrice N, Cohen $\mathrm{P}$ and Hemmings BA: Mechanism of activation of protein kinase B by insulin and IGF-1. EMBO J 15: 6541-6551, 1996.

38. Schmitz KJ, Wohlschlaeger J, Lang H, Sotiropoulos GC, Malago M, Steveling K, Reis H, Cicinnati VR, Schmid KW and Baba HA: Activation of the ERK and AKT signalling pathway predicts poor prognosis in hepatocellular carcinoma and ERK activation in cancer tissue is associated with hepatitis $\mathrm{C}$ virus infection. J Hepatol 48: 83-90, 2008.

39. Nakanishi K, Sakamoto M, Yamasaki S, Todo S and Hirohashi S: Akt phosphorylation is a risk factor for early disease recurrence and poor prognosis in hepatocellular carcinoma. Cancer 103: 307-312, 2005.

This work is licensed under a Creative Commons Attribution-NonCommercial-NoDerivatives 4.0 International (CC BY-NC-ND 4.0) License. 